\title{
¿Adiós a la economía política? Problemas de parámetros, métodos y direcciones en la configuración de los estudios culturales centroamericanos
}

\author{
A Goodbye to Political Economy? Parameter, Method, and Direction \\ Problems in the Configuration of Central American Cultural Studies \\ Adeus à economia política? Problemas de parâmetros, métodos e \\ rumos na configuração dos estudos culturais centro-americanos
}

\section{Marc Zimmerman}

UNIVERSITY OF ILLINOIS AT CHICAGO/ UNIVERSITY OF HOUSTON, ESTADOS UNIDOS

Profesor Emérito del Programa de Estudios Latinos y Latinoamericanos de la Universidad de

Illinois, Chicago y del Departamento de Lengas Modernas y clásicas en la Universidad

de Houston, Texas. PhD en Literatura de la University of California, San Diego. Es autor de Literatura y testimonio en Centro-América: Posiciones post-insurgentes (Casa Editorial de la Universidad Rafael Landívar, 2006), Literature and resistance in Guatemala:

Textual Modes and Cultural Politics from El señor presidente to Rigoberta Menchú. 2 vols. (Ohio University Press, 1995); con John Beverley, Literature and Politics in the Central American Revolutions (University of Texas Press, 1990), Defending their Own in the Cold. The Cultural Turns of U.S. Puerto Ricans (University of Illinois Press, 2011), U.S. Latino Literature (MARCH/Abrazo Press, 1992). Correo electrónico: mzimmerman@uh.edu

Ensayo

Versión revisada de una ponencia presentada en la Primera Conferencia de Estudios culturales centroamericanos en la Universidad de El Salvador, San Salvador, 2007, ya con unas referencias a acontecimientos más recientes que la fecha de autoría original. Documento accesible en línea desde la siguiente dirección: http://revistas.javeriana.edu.co doi: 10.11144/Javeriana.cl19-38.aplm

Cómo citar este ensayo:

Zimmerman, Marc. " ¿Adiós a la economía política? Problemas de parámetros, métodos y direcciones en la configuración de los estudios culturales centroamericanos". Cuadernos de Literatura 19.38 (2015): 34-43. http://dx.doi.org/10.11144/Javeriana.cl19-38.aplm 


\section{El problema teórico}

Desde la época de los movimientos revolucionarios, la cuestión de cómo estudiar las sociedades latinoamericanas se ha enfocado con más y más frecuencia en lo que antes se consideraban cuestiones secundarias o "superestructurales". Dentro de este contexto, los estudios culturales de una variedad u otra han pasado a un escenario céntrico en un esfuerzo por elaborar modelos teóricos y crear polémicas con respecto a asuntos cruciales para desarrollos futuros. Durante el mismo periodo, Centroamérica ha vivido una transición crucial que involucra procesos de paz y democratización en el contexto de la imposición de políticas económicas neo-liberales así como conflictos socio-políticos persistentes y crisis naturales más graves. Cuestiones con respecto a género, etnia, nuevas identidades e identificaciones nacionales; cuestiones de inmigración masiva, sobre crecientes olas de criminalizacion y violencia tanto como crecientes controversias sobre eco-política y mezclas culturales, son entre los asuntos más comunes. Estos habían sido minimizados en estudios previos de casi cada país o fenómeno latinoamericano. Pero las preguntas que surgen de las teorías de estudios culturales no pueden tener menos y de hecho - dado la gran debilidad y vulnerabilidad económica de la región- pueden tener más importancia en Centroamérica y especialmente en sus países norteños.

Conocemos las circunstancias y los ejemplos siempre citados: los sandinistas malinterpretaron los asuntos de los miskitu, impusieron su voluntad sobre los afro-caribeños de Bluefields, negaron agendas de género y las perspectivas feministas y las gay. El FMLN concibió mal las perspectivas y actitudes urbanas de su país. La izquierda guatemalteca hizo una mala lectura de las relaciones indígenas y por supuesto, las cuestiones guatemaltecas se extendieron a Chiapas en los años noventa como parte de un foco meso-latinoamericano más amplio que abarcó movimientos sociales y subalternos en las vísperas del neo-liberalismo y la globalización.

Algunas formas tempranas del marxismo explicaron las relaciones en función de luchas de liberación nacional en contra de relaciones feudales y neo-coloniales; la teoría de la dependencia enfatizó las luchas en contra del imperialismo y la dominación cultural en un sistema total en el cual el nacionalismo en sí era parte de una estructura de dependencia. El fracaso de Chile en los años setenta y de los movimientos revolucionarios de los ochenta, así como el colapso del movimiento internacional comunista y el desencanto con el experimento cubano, condujeron hacia una crítica de las ideologías de izquierda y del marxismo en sí. Estos se vieron como parte de un modo eurocéntrico colonizador inadecuado y hasta erróneo para gran parte de América Latina y del mundo entero. 
Los mismos movimientos revolucionarios dejaron de ser vistos como luchas bipolares entre el capitalismo y el socialismo y se convirtieron en intentos de última hora por parte de elites de oposición en contra de la ola inevitable de un sistema globalizante en el que las luchas principales involucraron grupos, configuraciones y bloques inestables y siempre cambiantes. Además los problemas con las perspectivas y acciones revolucionarias centroamericanas con respecto a asuntos indígenas, afro-caribeños y feministas condujeron al rechazo de previos modelos explicativos centrados en modos de producción, lucha de clase, imperialismo cultural y demás; y condujeron también al aumento de críticas que enfatizan combinaciones variantes de factores sociales, étnicos, religiosos, etcétera, en un proceso general de globalización en el cual luchas entre fuerzas locales y globales, fuerzas sectoriales y generales se agudizan, se contradicen y son difíciles de contener.

La irrupción de las aplicaciones a América Latina de la teoría post-colonial agudizó la distinción entre cuestiones teóricas y construcciones aplicables a diferentes partes del mundo colonizado. La pérdida de fe en la crítica marxista normativa condujo tanto a nuevas teorías no-economistas de fuerzas y movimientos sociales, como a nuevas orientaciones de la modernidad, gobernabilidad y la ingobernabilidad que posiblemente obtienen su formulación más radical en un tipo de crítica post-marxista que, originada en medio de la lucha postcolonial de la India, halló sus propias variantes y puntos de controversia entre los historiadores latinoamericanistas por un lado y los literatos de estudios culturales por el otro.

Dentro del nuevo ambiente intelectual el cambio ha sido en la dirección de Foucault, Baudrillard, Bourdieu, Stuart Hall, Laclau y Mouffe, Negri y Hardt. Otros se han basado en el trabajo de teóricos sociales como Brünner, Lechner y Calderón, y también en teóricos centrados en asuntos culturales como Martín Barbero, Richard, Sarlo y García Canclini. En la escena latinoamericanista de los Estados Unidos, Jean Franco, George Yúdice, Juan Flores y otros empezaron a introducir y elaborar una visión nueva, mientras figuras como John Beverley, Patricia Seed e Ileana Rodríguez empezaron por proponer cuestiones subalternas como reacción a las críticas dominantes. Al final sus discordancias son menos importantes que sus similitudes en el surgimiento de una crítica contemporánea que está a la vanguardia del pensamiento crítico en la era de la globalización, de los procesos transnacionales y movimientos sociales. Estas tendencias ahora se han juntado en la formación en la década de 1990 del Task Force de cultura, política y poder dentro de la Asociación de Estudios Latinoamericanos y la iniciativa de Cultura, política y poder dentro de la Asociación Internacional de Estudios Culturales. 
A pesar de todas estas re-orientaciones teóricas, había una ausencia sobresaliente en el desarrollo de la construcción teórica latinoamericana de estos días: precisamente una perspectiva sobre una de las áreas claves del hemisferio, cuyos fracasos revolucionarios abrieron la nueva época socio-política e ideológica-Centroamérica. De hecho estos influyeron mucho en la teoría post-marxista mientras que nuestra atención giraba después de Esquipulas II a NAFTA, Chiapas y a los nuevos procesos y luchas de democratización que han surgido en Venezuela, Bolivia, Ecuador, Argentina, Uruguay y otros lugares. Curiosamente entre la gran mayoría de los teóricos ya mencionados arriba, ha habido un gran silencio sobre los procesos culturales de América Central.

En el contexto actual nunca ha habido tanto interés en la literatura y cultura centroamericanas por especialistas no centroamericanos (el resultado de intereses y carreras que se desarrollaron en el período de crisis previo). En contraste el interés fuera del círculo de los especialistas ha sido poco. Hasta cierto punto, Centroamérica está aislada de nuevo y solo las controversias incitadas por libros como El asco de Horacio Castellanos Moya, Adiós, muchachos de Sergio Ramírez y El país bajo mi piel de Gioconda Belli, así como la controversia provocada por la apropiación de Mario Roberto Morales (1998) de las ideas de García Canclini para atacar a la política de identidad indígena en Guatemala -y sobretodo, la controversia Rigoberta Menchú / David Stoll- han mantenido a flote el interés en los estudios culturales centroamericanos (ver Stoll 1999; Arias 2001 y Zimmerman 2006). Sin embargo, tales debates se han mantenido distantes de las teorías de transición socio-económica y política, por ejemplo la teoría de John Booth y Thomas Walker de cambio de régimen, presentada en la tercera edición de su libro sobre Centroamérica (1999). Y eso es especialmente serio porque todo el trabajo clave sobre Centroamérica demuestra que no podemos entender procesos socio-económicos y políticos sin fijar sus conexiones más profundas con las dimensiones del desarrollo cultural, artístico e ideológico.

El campo de estudios culturales centroamericanos halla su formulación inicial más importante en los debates sobre el marxismo ortodoxo de La patria del criollo de Severo Martínez Peláez (1970) y los textos gramscianos de Sergio Ramírez; empezando con Balcanes se desarrolla y se elabora en los años ochenta y más allá en los estudios del populismo, indigenismo, religión, cultura popular, estructura familiar, vida cotidiana, género y "estudios queer", entre otros temas ,así como en los trabajos de Roger Lancaster, Erick Blandón-Guevara, Carlos Vilas, Kay Warren, Diane Nelson, Marta Casaus, Emilio del Valle Escalante y otros. Ahora los debates mencionados, tanto como los nuevos libros de García Canclini y compañía, se unen a otros esfuerzos como la colección coordinada por Mabel 
Moraña (2000), y la colección editada por Ana del Sarto, Alicia Ríos y Abril Trigo (2004) para poner en relieve un campo de trabajo que debe fortalecer los estudios culturales latinoamericanos y centroamericanos en los próximos años, especialmente en relación con las nuevas fuerzas de globalización y transnacionalización.

El propósito de este ensayo es contribuir a este desarrollo, pero siempre con cierto grado saludable de escepticismo con respecto a los modelos iniciales de estudios culturales, especialmente el concepto de construcción social, como lo proponen Stuart Hall y otros intelectuales de la segunda generación de la escuela de Birmingham, tanto como sus seguidores en estudios postmarxistas, postmodernos, postcoloniales y subalternos en América Latina.

La deuda que tienen los estudios culturales latinoamericanos con los estudios culturales generales debe ser vista en relación a su distanciamiento teórico de las categorías tradicionales para que las perspectivas sobre cuestiones de etnia, género, sexualidad, etcétera, puedan encontrar un papel más determinado y específico en la articulación de discursos. La palabra "articulación" es la conexión clave con la cual los latinoamericanistas y en nuestro caso, los centroamericanistas, intentan captar las particularidades y especificaciones de su formación social variante. La meta de los estudios culturales centroamericanos "en vías de desarrollo" debe ser la de explorar las dimensiones hemisféricas y regionales necesarias para constituir una versión local apropiada, adecuada y fructífera para examinar el pasado, el presente y el futuro de la realidad centroamericana.

En el proceso se debe especificar y diferenciar nuestro acercamiento con relación a aquellas construcciones de estudios culturales que dependen demasiado de una teoría de construcción social y discursiva. Mientras que es una dimensión importante, también ha sido exagerado y convertido en el sine qua non de los estudios culturales, como si no hubiera una realidad fuera de su construcción discursiva e ideológica. En este contexto es importante considerar la cuestión que Daniel Mato y otros han formulado recientemente: $\dot{\mathrm{d}}^{\text {se }}$ debe distinguir entre estudios culturales latinoamericanos desarrollados en el mundo de habla inglesa y la forma que toma en el terreno latinoamericano? Esta cuestión está enraizada en la notoria despoliticización y en el elitismo de los estudios culturales en los Estados Unidos en comparación con la versión necesariamente politizada de estudios culturales que, centrada sobre relaciones de poder, emerge en Mato y otros en el campo latinoamericano.

En un esfuerzo por encontrar una base diferencial para la inserción de América Latina en los estudios culturales a nivel global, Mato, como coordinador de la Asociación Latinoamericana de Cultura, Política y Poder, reconoce la deuda teórica de los estudios culturales latinoamericanos a sus antecedentes británicos 
(Mato 2002). Sin embargo, quiere hacer una distinción sumamente aguda entre las versiones británicas y estadounidenses de estudios culturales latinoamericanos por un parte y por otra, lo que él considera las dimensiones fundamentales de la teoría de estudios culturales desarrollándose actualmente dentro de América Latina. De hecho Mato enfatiza el hecho de que América Latina tiene sus propias tradiciones específicas de crítica cultural y que supuestas influencias de París o Birmingham son paralelos (y no préstamos) que se reconocían retrospectivamente y con frecuencia.

Mientras una figura como Jesús Martín Barbero puede citar un sinnúmero de antecedentes europeos, su trabajo y el trabajo de sus colegas famosos se desarrolló dentro del marco de tradiciones netamente latinoamericanas. Además y más importante para Mato, los estudios culturales en América Latina tienen que distinguirse de sus "primos" en Birmingham y los practicantes en Estados Unidos, con la designación de Latin American Studies in Culture and Power, indicando de esta manera su énfasis firme en las dimensiones políticas de las prácticas culturales y en los efectos de poder inherente en, y emanando de, esas misma prácticas culturales. Esta dimensión, insiste Mato, marca la diferencia más evidente entre los estudios culturales latinoamericanos y aquellas versiones que él ve como céntricas en los Estados Unidos y de hecho, en las últimos giros teóricos del trabajo de Stuart Hall.

Aunque Mato da privilegio epistemológico a los intentos teóricos realizados en América Latina, tales re-orientaciones pueden conllevar su propia ceguera: una observación irreflexiva y demasiado conveniente que obvia los prejuicios y prácticas residuales criollistas, subestimando así, y como dirían Aníbal Quijano y Walter Mignolo, "la colonialidad de poder" que persiste en los intersticios de tales discursos y sus presuposiciones teóricas. A mi ver, Mato se inclina demasiado hacia una esencialización de la latinoamericanidad, aunque niega esa posibilidad; y es cierto que las divisiones recientes entre las perspectivas de los estudios culturales versus las de los estudios subalternos, son como una suerte de guerra clasista y una lucha para controlar el terreno. Sin embargo, se supone que persiste un sentido de comunidad política (tal vez imaginaria) que se basa en una oposición mutua a la hegemonía neoliberal y sus verdugos trans-continentales.

El nuevo orden capitalista exige y crea procesos de inestabilidad, ingobernabilidad, fragmentación, desorden y hasta oposición para su propia supervivencia y expansión. Pero el sistema está así haciendo que proliferen procesos que bien pueden girar de manera decisiva en su contra, creando nuevos espacios en los cuales la oposición pueda erigir formas de resistencia subalterna cada vez más activas, poderosas y a veces capaces de liberarse. Esta es una dimensión clave del 
nuevo (des)orden del mundo realizada en los últimos años con los giros variados hacia la izquierda en varios países del hemisferio (ver Zimmerman y Balboa, 2012).

\section{Los estudios subalternos y su estatus en el juego teórico}

Las perspectivas subalternas empezaron a contribuir al desarrollo y profundización de nuestro entendimiento de la construcción y reproducción extendida de la estigmatización y la marginalización en el mundo contemporáneo. Sin embargo, implícita en esta consideración está la cuestión de la representación, o sea ¿quién tiene el poder legítimo de hablar por los subalternos y de qué manera es la autoridad de hacerlo construida discursivamente? Aquí las apelaciones a la heterogeneidad y a la hibridez no son completamente satisfactorias. Lo que es central para este asunto son las cuestiones sobre el estado de los intelectuales subalternos que desafían los viejos modelos. Hoy los estudios subalternos involucran una crítica postcolonial de las teorías y teóricos viejos y nuevos en su capacidad de captar los viejos y nuevos movimientos sociales tomando en consideración las particularidades de los casos latinoamericanos y sin esencializar, romantizar u homogenizar la conciencia subalterna.

Los estudios subalternos latinoamericanos se postulan como una crítica radical de las versiones izquierdistas de los estudios culturales. Sin embargo hay problemas. Aun si se acepta la necesidad de captar el discurso subalterno, ¿̇cómo pueden los críticos latinoamericanistas radicales -quienes son subalternos en sí mismos solamente en relación a las normas críticas hegemónicas- estar seguros de su propia gnosis como base adecuada de su praxis? ¿Cómo podemos desarrollar una crítica de la epistemología subalterna? ${ }_{\mathrm{C}}$ Es factible? Y si podemos elevar la cuestión de la legitimidad de todas las formas de conocimiento, ¿de qué manera y de acuerdo con qué criterios podemos establecer parámetros más o menos razonables para nuestro campo de estudio? ${ }_{\mathrm{C} P o d e m o s}$ vencer nuestra tendencia a homogenizar o romantizar la conciencia subalterna? ¿Qué pasa con nuestra tendencia a privilegiar el discurso indígena, minoritario o subalterno, $o$ cómo estar seguros de que lo hemos hallado cuando existe la posibilidad de que es otra forma de ventriloquia colonial? ¿Cómo podemos superar la idea de que los oprimidos son de alguna manera necesariamente virtuosos?

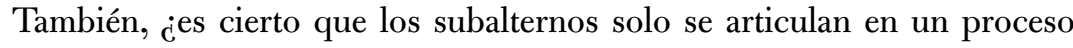
que termina cuando se finaliza su estado de subalternidad? $\mathrm{O}$ iel grado en el que los subalternos ya participan es un esfuerzo público capaz de totalizar todo? ${ }_{\mathrm{C}}$ Es verdad que detrás de estos grupos étnicos y movimientos sociales están las grandes fundaciones internacionales? ¿Sin las fundaciones, tendrían los grupos algún impacto? ¿Quiénes son los Che Guevaras o tupamaros de la postmodernidad 
globalizada, o se hace innecesaria una vanguardia heroica dentro de aquélla? En fin, ${ }_{\mathrm{i}}$ Son los estudios subalternos simplemente la tropicalización del Sur de parte de los teóricos del Norte? ¿Es posible que (por fin) el pensamiento desde el Sur sea capaz de transformar el pensamiento del Norte? Estas son las cuestiones que emergen en la crítica que hizo George Yúdice (2001) del análisis de John Beverley (1999) de las teorías del consumo en Néstor García Canclini (1995). Surgen también en la colección editada por Jon Beasly-Murray y Alberto Moreiras (2001) sobre la subalternidad latinoamericana, donde empezamos a ver una ruptura inclusive con respecto a Antono Gramsci, unos de las figuras claves para el pensamiento marxista y pos-marxista de América Latina ${ }^{1}$. Estas son las interrogaciones cuyas respuestas nos esperan mientras el siglo nuevo va por su propio rumbo.

\section{Preguntas finales}

Claro, no se puede pensar lo cultural en Centroamérica sin pensar su situación historico-social, los proyectos de modernizacion al nivel económico tanto como cultural. Y no es que se tiene que mencionar lo económico ni mucho menos la economía política sino que está ahí por todas partes. Es obviamente imposible tratar el tema cultural sin trazar su conexión necesaria, clara o fundamental con lo económico. En los estudios recientes tenemos mucho sobre historia y memoria cultural, sobre realidad y representación indígena y caribeña, cultura y democracia, posmodernidad y modernidad, sobre la ingobernabiliidad, sobre la inmigración, sobre la mara trucha, la Bestia y los demás. Pero en todo eso, parece como la famosa "superestructura" vuelve a ser más clave que la famosa "infraestructura" marxista hasta que nos hace preguntar si las nuevas circunstancias en la región no implican, hasta cierto punto por lo menos, que debemos decir adiós a la economía política como clave para el análisis de los asuntos centroamericanos.

Pero aquí enfrentamos el cunundrum, el catch 22. Porque tumbando viejos paradigmas, ¿dónde estamos? Porque si no domina lo económico, ¿qué domina? Es una combinación de fuerzas, contestaría uno. Pero ¿ ¿bajo qué condiciones

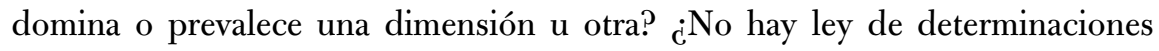
cambiantes? ¿ Todo es puro caos y empiricismo? ¿ Como podemos medir la importancia de cualquier cosa que estudiamos? ¿Con qué instrumentos podemos prender un análisis que tiene ciertas bases de validez? ¿Cómo pensamos la región cuyos problemas profundos queremos entender y resolver? Así que tan fácil es decir adiós, pero ¿cómo y a qué decimos hola?

1 Esta tendencia se culminó con la publicación del libro de Jon Beasly-Murray (2010). 


\section{Obras citadas}

Arias, Arturo, ed. The Rigoberta Menchu Controversy.

Minneapolis: University of Minnesota Press, 2001.

Baeza Ventura, Gabriela y Marc Zimmerman, coords. Estudios culturales

centroamericanos en el nuevo milenio. San José, C.R.:

Editorial de la Universidad de Costa Rica, 2009.

Beasly-Murray,Jon. Posthegemony: Political Theory and Latin America.

Minneapolis. University of Minnesota Press, 2010.

Beasly-Murray, Jon, y Alberto Moreiras, eds. "Subalternity

and Affect". Angelaki 6.1 (2001): 1-4.

Belli, Gioconda. El país bajo mi piel. Memorias de amor

y guerra. Barcelona: Seix Barral, 2005.

Beverley, John. Subalternity and Representation: Arguments in

Cultural Theory. Durham: Duke University Press, 1999.

Booth, John A., Christine J. Wade, y Thomas W. Walker. Understanding Central

America: Global Forces, Rebellion, and Change. Boulder: Westview Press, 1999.

Castellanos Moya, Horacio. El asco. Barcelona: Tusquets, 2007.

del Sarto, Ana, Alicia Ríos y Abril Trigo. The Latin American Cultural

Studies Reader. Durham,N.C.: Duke University Press, 2004.

García Canclini, Néstor. Consumidores y ciudadanos. México: Grijalbo, 1995.

Martínez Peláez, Severo. La patria del criollo. Guatemala: Editorial Universitaria, 1970.

Mato, Daniel, "Estudios y otras prácticas intelectuales latinoamericanas

en cultura y poder", en Daniel Mato (coord), Estudios y Otras

Prácticas Intelectuales Latinoamericanas en Cultura y Poder.

Caracas: Consejo Latinoamericano de Ciencias Sociales (CLACSO)

y CEAP, FACES, Universidad Central de Venezuela, 2002.

Morales, Mario Roberto. La articulación de las diferencias o el Síndrome de Maximón. Ciudad de Guatemala: FLACSO, 1998.

Moraña, Mabel. Nuevas perspectivas desde/sobre América Latina: El desafío de los estudios culturales. Santiago de Chile: Instituto de Literatura Iberoamericana, Editorial Cuarto Propio, 2000.

Ramírez, Sergio. Adiós, muchachos. Madrid: Alfaguara, 2007.

-. Balcanes y volcanes y otros ensayos y trabajos. Buenos Aires: Editorial Nueva América, 1985.

Stoll, David. Rigoberta Menchú and the Story of All Poor

Guatemalans. Boulder: Westview Press, 1999.

Yúdice, George . "Civil Society, Consumption, and Governmentality in an Age of Global Restructuring: An Introduction". Social Text 45 (1995): 1-25. 
-. El recurso de la cultura. Barcelona: Paidós, 2001.

Zimmerman, Marc. Literatura y testimonio en Centro-América:

Posiciones post-insurgentes. Cuidad de Guatemala: Casa

Editorial de la Universidad Rafael Landívar, 2006.

Zimmerman, Marc y Luis Ochoa Bilbao, eds. Giros culturales en la marea rosa de América Latina. Puebla, México: Benemérita Universidad Autónoma de Puebla (BUAP) y LACASA, 2012. Republicado en Alter/nativas Latin American Cultural Studies journal (Online Book series). Ohio:

State University. Disponible en http://alternativas.osu.edu/assets/ files/ZIMMERMAN-OCHO-Marea-Rosa-completa-final.pdf. 